\title{
Digital measurement of operant disk press force maintained in CD-1, BALB/c, and C57BL/6 mice
}

\author{
TROY J. ZARCONE and STEPHEN C. FOWLER \\ Life Span Institute, University of Kansas, Lawrence, Kansas
}

\begin{abstract}
Force of disk press responses by inbred (C57BL/6 and BALB/c) and outbred (CD-1) mice were measured with a PC/DOS-based system, which allowed for continuous measurement of pressing, as well as for control of reinforcer presentation on the basis of response-force dimensions. Photobeam-based measurement of mouse entry into a hopper where reinforcers were presented provided additional information about anticipatory and consumatory behavior in relation to environmental stimuli leading up to reinforcer delivery. Disk pressing was generated and measured with the use of an analog-to-digital interface with all three mouse strains. The strains differed in the physical and temporal characteristics of the disk press, as well as in the behavioral chain leading to reinforcer presentation. These measurement methods appear well suited for quantitating functional behavioral differences occasioned by genetic variations in mice.
\end{abstract}

Interest in the physical characteristics of operant behavior began with Skinner's (1938) initial description of response force during leverpressing performance. Skinner measured the "intensity" (i.e., force) and duration of responses by attaching a ballistic pendulum with adjustable electrical contacts and a Telechron clock to the response lever and recorded these response dimensions on a special kymograph (Skinner, 1938, p. 310). Interest in the physical characteristics of responses was pursued by Notterman and colleagues (e.g., Notterman \& Mintz, 1965), who were the first to use a force transducer (based on the linear variable differential transformer principle) to measure the operant response of rats. The Notterman lab was also the first to use a computer (an analog computer) to process these force-proportional voltage signals to produce measures of individual responses (e.g., peak force, duration, and time integral of force).

Fowler continued by applying new technologies to the measurement of individual response characteristics of operant behavior (Fowler, 1974; Fowler \& Leberer, 1977) and more recently described an Apple II, IIe microcomputer system for conducting operant research (Fowler, 1985). Since the Apple-based system is now obsolete, one purpose of this paper is to describe a DOS/PC-based system that implements the measurement concepts defined in previous research (Fowler, 1974, 1985; Notterman \& Mintz, 1965). The other purpose of this paper is to illustrate the extension of this technology to the analysis of operant disk pressing by inbred and outbred strains of mice. Behavioral genetic research with mice has encoun-

This research was supported by Grants DA 12508 and MH 43429 We thank Rong Chen for her assistance in the collection of these data. Correspondence concerning this article should be addressed to T. J. Zarcone, 1001 Sunnyside Ln., 1052 Dole, Life Span Institute, Lawrence, KS 66045 (e-mail: tzarcone@ku.edu). tered difficulty in replicating some, but not all, effects across laboratories (Crabbe, Wahlsten, \& Dudek, 1999). This problem may be partially due to the extensive amount of experimenter handling needed to run mice through many of these learning tasks. The procedures, apparatus, hardware, and software described in this paper may provide a solution to some of these problems by limiting the impact of experimenter influences on mouse performance.

\section{METHOD}

\section{Subjects and Materials}

The mice (1 CD-1, 1 BALB/c, and 1 C57BL/6, Charles River Laboratories) used in these demonstrations were taken from an ongoing experiment supported by the National Institute on Drug Abuse (R21 DA12508). Operant chambers, each enclosed in a separate sound-attenuating box with exhaust fan, were customized to measure disk pressing by mice. An intelligence panel was mounted on the front of each mouse chamber $(23.5 \times 21.5 \times 18.5 \mathrm{~cm})$. The houselight at the top of the panel contained a white $24-\mathrm{V}$ bulb (GE 1219) mounted behind a translucent Plexiglas cover. A 24-V, 2900-Hz sonalert piezoelectric buzzer (Kayer, 11S3240TA) was connected to the houselight and mounted to the right of the houselight behind the intelligence panel. The sonalert generated a 70-db tone whenever the houselight was turned on. The reward hopper (5.5- $\mathrm{cm}$ diameter, $0.2 \mathrm{~cm}$ from bottom of cage) was located below and to the right of the houselight. A photo microsensor (Omron, EESPW 301, \$60) detected entries into and withdrawals from the reward hopper. The beam was located $1 \mathrm{~cm}$ inside and $1 \mathrm{~cm}$ from the bottom of the hopper and ran horizontal and parallel to the intelligence panel. An electromechanical dipper, designed for rats (Gerbrand, G5600 GS-RH, \$400) and mounted just behind the reward hopper and outside the cage, presented $0.05 \mathrm{ml}$ of sweetened condensed milk for $5 \mathrm{sec}$ into the reward hopper via a hole located $3 \mathrm{~cm}$ deep in the hopper. A baffle (a rounded, L-shaped piece of aluminum that blocked the top half and end of the hopper) was added to adapt the hopper to mice (i.e., the baffle reduced the hopper space so that only a mouse's anterior half could enter the hopper).

A $0.5-\mathrm{cm}$ diameter hole was located $15 \mathrm{~cm}$ below the houselight and $0.5 \mathrm{~cm}$ from the cage floor. A force-sensitive disk was recessed 
$0.2 \mathrm{~cm}$ behind the hole and was silent and isometric. Our particular sensors were based on a Model 31 Sensotec (Columbus, OH) load cell with a $0-250 \mathrm{~g}$ range $(\sim 750)$ capable of measuring both tension and compression load forces (see www.sensotec.com for additional specifications). In the present application, the disk-load cell was oriented behind the intelligence panel so that only compression force could be applied. The signal generated by the sensor was powered and amplified by a signal conditioner/amplif ier with integral power supply unit (Sensotec SA-4, 060-3148-06, 5VDC excitation; Sensotec Model GM, $\sim \$ 450$, is a replacement). Each transducer signal was connected to a separate analog input channel of a Labmaster DMA (Scientific Solutions, 938193) interface card $(\sim 1,000)$. Other, custom-built, interfacing served the experimental chambers via a 486 DX2 PC computer and provided the control for the houselight, sonalert, and dipper while collecting data from the force-sensing disk and the hopper photo microsensor. Data for each mouse were written to RAM and then transferred to the hard drive at the end of each session. The computer, with appropriate peripherals, including an inkjet printer and a 100-megabyte Zip drive, served all eight chambers simultaneously. The software controlling these chambers was written by the authors in Turbo Pascal 4.0.

\section{Procedure}

Daily sessions were divided into three distinct periods marked by both auditory and visual stimuli. Each period was signaled by a change in the illumination of the houselight and operation of the sonalert. The purpose of these periods was to consistently schedule changes in environmental stimuli (light and tone off to light and tone pulsing) that preceded a stimulus condition (light and tone continuously on) in which disk presses were followed by the presentation of sweetened condensed milk. That is, the stimuli were intended to signal that food would be available in several minutes but was not available now. The houselight and sonalert were off during the first period (Period 1, $10 \mathrm{~min}$ ), and disk presses were not followed by milk presentation. The houselight flashed and the sonalert beeped ( $1 \mathrm{sec}$ on/1 sec off) during the second period (Period 2, $10 \mathrm{~min}$ ), and disk presses were not followed by milk presentation. The houselight and the sonalert remained on constantly for the remainder of the session $(30 \mathrm{~min}$ and $30 \mathrm{sec}$ ). Access to the reward (1 part Borden's sweetened condensed milk and 2 parts tap water) did not occur until $30 \mathrm{sec}$ after the start of the constant light and sonalert stimuli that signaled that Period 3 was operative. A 30-sec delay, or hold interval, was added in order to reduce the potential for adventitious reinforcement of disk presses or hopper entries that might occur at the end of Period 2. Data from the hold interval were analyzed separately from the data collected from the three periods.

Disk press training consisted of baiting the disk with milk smeared onto the disk with a milk-saturated cotton swab. With this procedure, disk presses quickly came under control of the reinforcement contingency of fixed ratio 1 for presses greater than $2 \mathrm{~g}$. After responding was determined to be stable via visual inspection of the number of disk presses during the session, the response peak force requirement was changed to $4 \mathrm{~g}$ and then to $8 \mathrm{~g}$. Milk was delivered upon response termination, defined by the force's falling below $2 \mathrm{~g}$ after reaching its peak amplitude.

\section{Data Collection}

The force-time waveform data produced by presses to the disk were measured with the Labmaster at 100 samples/sec. Separate quantities of disk pressing were computed (e.g., peak force, duration) during the experiment (i.e., on line) and in real time (see Fowler, 1987, for a description of measurement concepts). The online, real-time data were then used to program reinforcer presentations based on peak force. The occurrence of events produced by entries into the hopper was measured (also with 0.01-sec resolution) with the digital I/O section of the Labmaster. Disk presses and hopper entry durations had to be at least $0.02 \mathrm{sec}$ to be defined as a response. This temporal definition was added in order to filter out occasional (e.g., once every third or fourth session) spikes (i.e., electrical noise) that occurred in the signal. These spikes were measured and archived with the raw data files (see below) but did not influence the contingencies during the session and were filtered in the data analysis software (see the Appendix). The presence or absence of hopper entries can also be used to program environmental events but was not used for such purposes in the present demonstration.

Data were archived into integer and text file formats. The integer file recorded a force value every $0.01 \mathrm{sec}$. Head entries were incorporated into this array by replacing the force value with a dummy value of -20 . This arrangement made it possible to use artificially generated negative values (e.g., -20) to record hopper entries in the single column data stream of the integer file (i.e., raw data file). Other events that were mutually exclusive to disk pressing can be measured in a similar fashion. This system provided a simple archival format that allows for a complete reconstruction of the disk and hopper events during a session. Separate summary text files of disk presses and hopper entries were made for initial off-line data analyses. Summary disk press text files consisted of a matrix of timestamped disk press events (based on time since the beginning of a session). Measured parameters for these events included individual peak force, time integral of force (measure of effort), response duration, time since last response (interresponse time, IRT), and whether the response resulted in a reinforcer presentation (cf. Fowler, 1974). Summary hopper entry text files consisted of a similar time-stamped matrix and included the duration of the hopper entry and the IRT from the last hopper entry. An additional printed summary was generated at the end of each session. Integer and text data files were archived on write-only CD ROM disks.

\section{Data Analysis}

The software to analyze raw files and summary text files were written by the authors using Visual Basic 6.0. Raw data files were searched for specific behavioral events-for example, the end of disk presses or the beginning and end of hopper entries (see the Appendix for Visual Basic 6.0 code samples). When a behavioral event was detected, it was added to a variable, and timing and force dimensions were calculated and saved to other variables. These data were then summarized by period $(1,2$, hold, and 3$)$ and formatted into text files for export into Sigmaplot 2000 for Windows and other analysis software packages.

\section{Results}

Figure 1 shows samples of the event and force records for an outbred mouse (CD-1 \#1; top six panels) and for two inbred mice (C57BL/6 \#3; middle six panels; BALB/c \#1, bottom six panels), respectively. Data were sampled from the last session of the second exposure to the 8 -g force requirement condition (Session \#78), after exposure to other force requirement conditions (i.e., 2, 4, 8, 16, and $32 \mathrm{~g}$ ). Raw data were transformed from integer files to ASCII files using Visual Basic 6.0 written by the authors and imported into SigmaPlot 2000. Data are shown for the last $30 \mathrm{sec}$ of Period 1 (no food, dark-silent), the first $30 \mathrm{sec}$ of Period 2 (no food, pulsed light and sound), the last $30 \mathrm{sec}$ of Period 2, all of the 30-sec hold, and the first $30 \mathrm{sec}$ of Period 3 (food, light and sound on). The bottom four graphs of each group expand the time scale of the force records (top graphs of each group) for the sections highlighted by light gray boxes. Downward deflections of the event line (dark gray line) to HE (hopper entry) show when a mouse broke the photobeam located 


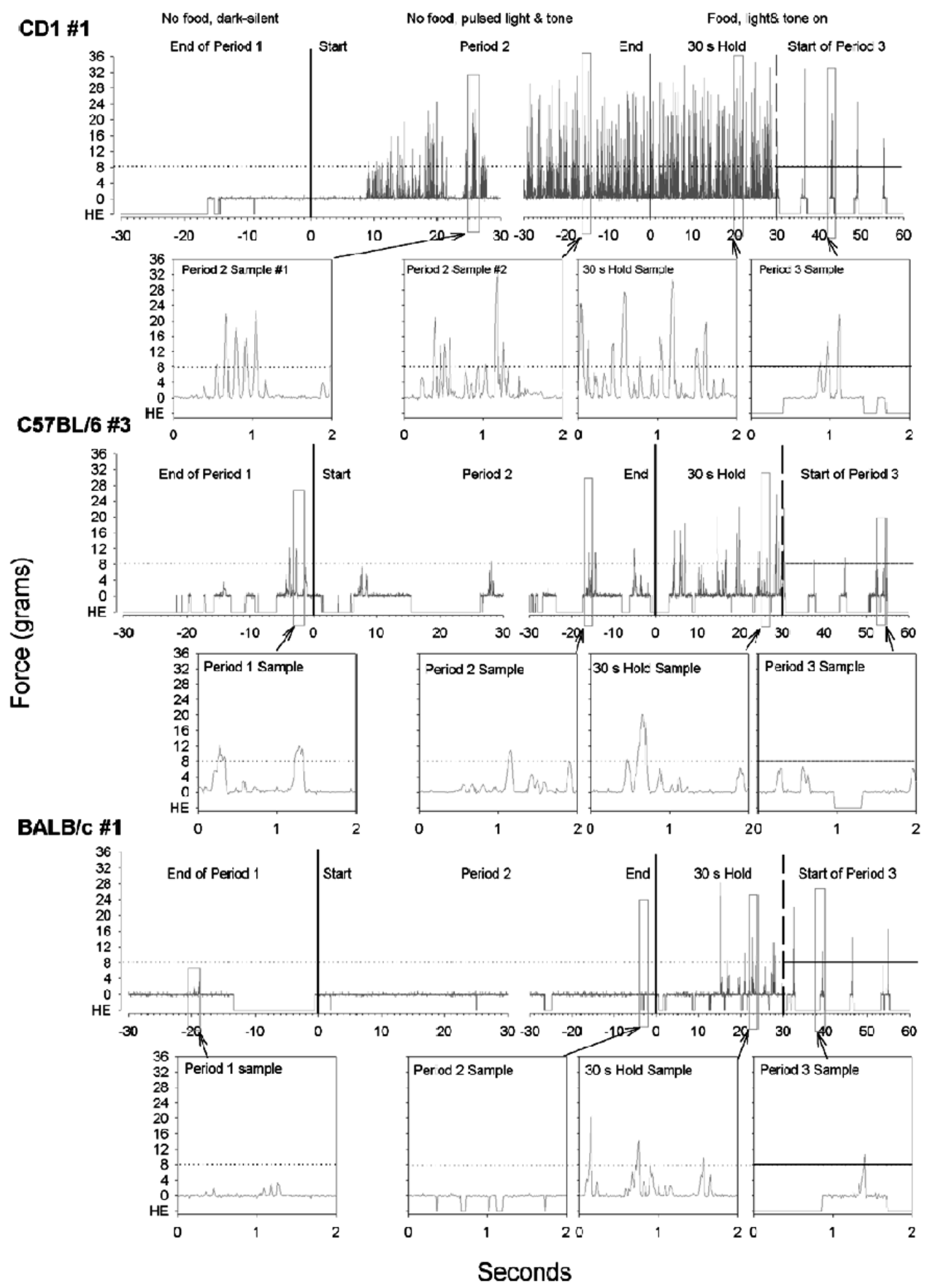

Figure 1. Samples of the disk press and food hopper event records grouped by mouse. The top six graphs show data for mouse CD1 \#1, the middle six graphs show data for mouse C57BL/6 \#3, and the bottom six graphs show data for mouse BALB/c \#1. Samples are shown for the last 30 sec of Period 1 (no food, dark-silent), the first 30 sec of Period 2 (no food, pulsed light and tone), and the last 30 sec of Period 2, all of the hold and the first 30 sec of Period 3 (food, light and tone on). The bottom four graphs temporally magnify the sections highlighted in the event record (light gray boxes). Deflections of the event line to hopper entry (HE) show when a mouse broke the photobeam located in the dipper hopper. The dotted and solid lines at $8 \mathrm{~g}$ on the $\boldsymbol{y}$-axis show the reinforcement criterion in effect only during Period 3. 


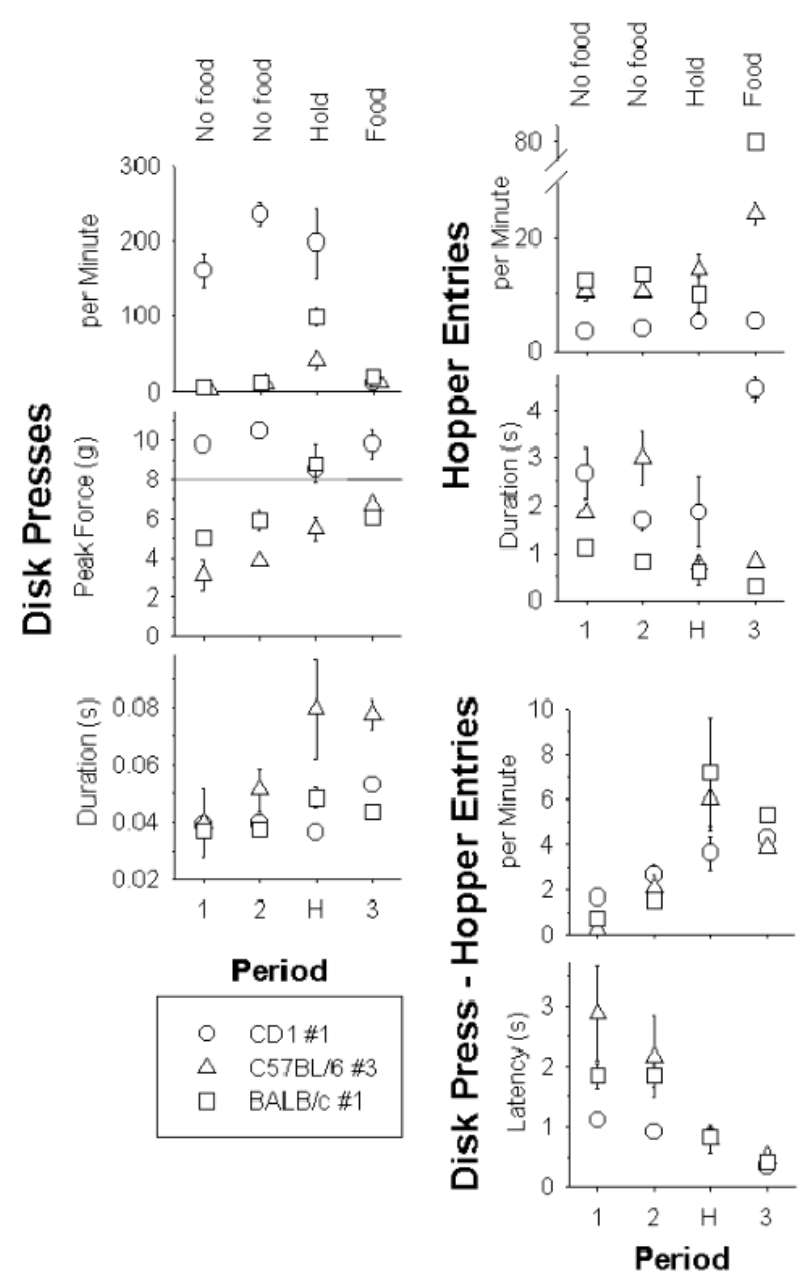

Figure 2. Summary data for mice CD1 \#1 (circles), C57BL/6 \#3 (triangles), and BALB/c \#1 (squares) for disk presses (rate, peak force, and duration), hopper entries (rate and duration), and disk press-hopper entry (rate and latency) behavior chain as a function of the anticipatory (Periods 1 and 2 and hold) and consummatory phases (Period 3 ). The dotted and solid lines at $8 \mathrm{~g}$ on the $\boldsymbol{y}$-axis for the peak force graph indicate that the reinforcement criterion was in effect during Period 3 only. Note the break in the $\boldsymbol{y}$-axis for the hopper entry per minute graph (top graph, right column).

in the dipper hopper. The dotted line at $8 \mathrm{~g}$ on the $y$-axis shows the reinforcement criterion that was effective only in Period 3 (shown with a solid black line).

These event samples reveal differences in disk pressing both within a session (across periods) and between mice. The event record samples for mouse CD-1 \#1 show that disk presses during the anticipatory periods (Periods 1, 2, and hold) occurred in "bouts." Many of these responses resulted in peak forces greater than the reinforcer requirement. No experimenter-controlled stimuli were presented during these periods to signal that the force criterion had been met, even though there were no experimenter provided stimuli that this criterion had to be met. During the consumatory phase (Period 3), disk pressing occurred in relatively smaller bouts that were followed by hopper entries. These data show that the onset of disk presses (slope of the force-time record) was steep and relatively constant up to the peak force. The termination of the disk press was also steep but occasionally marked with a slight increase in force (i.e., a hitch) toward the end of the press (Period 2 Sample \#2 and 30-sec hold sample). The shapes of the force-time recordings for the CD-1 mouse were similar to the shapes of the forelimb force recordings observed for rats (Fowler, 1987; Notterman \& Mintz, 1965). The event samples for mouse C57BL/6 \#3 also show disk pressing occurring at a lower peak force and with fewer disk presses per bout than that for mouse CD-1 \#1. During Period 3, disk pressing for mouse C57BL/6 \#3 occurred in relatively smaller bouts, and peak forces were much closer to the reinforcement criterion than peak forces for the CD-1 mouse. The expanded time scale samples show that the rise to peak force was not as steep as that for the CD-1 \#1 mouse. Mouse C57BL/ 6 \#3 also showed longer holds at the peak of the press, sometimes resulting in two peaks or notched waveforms before the force returned to zero. The declining slope of the forcetime curve leading to termination of the response was similar to that of mouse CD-1 \#1. The Period 3 sample for mouse C57BL/6 \#3 shows an instance of a hopper entry after the completion of a disk press that did not meet the reinforcement criterion. The event samples for mouse $\mathrm{BALB} / \mathrm{c}$ \#1 show disk pressing in all periods occurring at a lower rate than for mouse CD-1 \#1. During Period 3, disk pressing for mouse BALB/c \#1 occurred in relatively smaller bouts, as compared with the preceding phases, similar to that of mouse CD-1 \#1. The force-time waveforms for mouse BALB/c \#1 show that during the force rise to the peak, nonmonotonicities were sometimes present.

Figure 2 shows summary data averaged for each mouse across five consecutive sessions of the first exposure to the 8-g requirement condition (Sessions 44-48) for each dependent variable calculated from the raw data files using a Visual Basic 6.0 program written by the authors. The three graphs in the left column show parameters of disk pressing (response rate, peak force, and response duration) as a function of within-sessions stimulus conditions. The upper two graphs of the right column show parameters related to hopper entries (response rate and duration), and the bottom two graphs in the right show parameters related to the disk press-hopper entry behavior chain (response rate and latency between disk press and hopper entry). The top left graph of Figure 2 shows disk presses per minute, with disk presses defined as force above $2 \mathrm{~g}$ for at least $0.02 \mathrm{sec}$. Mouse CD-1 \#1 showed the highest rate of disk pressing during the first two periods and the hold. Reinforcer presentations (Period 3) resulted in equivocal response rates for the three mice. The middle graph on the left shows the peak force parameter of disk presses, which measures the highest force generated during a discrete press as defined above; the solid horizontal line shows that the 8 -g reinforcement crite- 
rion was in effect only during Period 3. Mouse CD-1 \#1 produced average peak forces greater than the reinforcement criterion during all conditions. Mice C57BL \#3 and BALB/c \#1 produced averaged peak forces lower than the reinforcement criterion during the first two periods. All three mice showed an increase in average peak force from Period 1 to Period 2. The bottom graph of the left column shows the mean duration of disk presses and was defined from the point at which the force exceeded $2 \mathrm{~g}$ until the force fell below $2 \mathrm{~g}$. During Period 1, all 3 mice produced similar average disk press durations. During the 30 -sec hold condition, the press durations for mice C57BL/6 \#3 and BALB/c \#1 increased.

The top graph of the right column shows the rate of hopper entries, which were defined by a photobeam break longer than $0.02 \mathrm{sec}$. Hopper entry rate was low during all conditions for mouse CD1 \#1. Mice C57BL/6 \#3 and $\mathrm{BALB} / \mathrm{c} \# 1$ had higher hopper entry rates than did the CD-1 \#1 mouse during Periods 1 and 2 and the hold condition. Both inbred mice showed an increase in hopper entry rate during Period 3 , with mouse BALB/c \#1 showing an increase of up to 80 responses per minute. The second graph for the hopper entry parameters shows the duration of hopper entries as defined above. There were no systematic changes across mice during Periods 1 and 2 and the hold on this measure. However, in Period 3, mouse CD-1 \#1 showed a dramatic increase in hopper entry duration with the addition of reinforcer presentations.

The bottom two graphs of the right column show parameters for the disk press-hopper entry chain. This response chain was defined as a disk press followed by a head entry with a latency of less than $5 \mathrm{sec}$. The per Minute graph for the rate of this response chain increased for all mice across Periods 1 and 2 and the 30 -sec hold condition. Response chain rates during Period 3 were similar for the 3 mice at around five responses/minute. The latency between a disk press and a hopper entry showed a decrease from Periods 1 and 2 to the 30 -sec hold condition, with an additional decrease during Period 3.

\section{DISCUSSION}

The force-based operant-conditioningchamber allowed for the quantitative measurement of disk pressing by mice from two different genetic strains and an outbred stock. This system also provided for on-line control of environmental stimuli in relation to the physical parameters of the disk press. Although the present study showed how reinforcer presentations could be made contingent on the peak force of a press to the disk, the measurement system also has the capability of making reinforcement delivery contingent on other behavioral parameters, such as hopper entry (or conjoint criteria).

One feature of the present system is that a record is created for the entire session at a resolution of $0.01 \mathrm{sec}$. This affords the experimenter the opportunity to reexamine relationships among variables off line after the experiment has been concluded. For example, the present experiment defined a disk press-hopper entry chain as a disk press followed by a hopper entry with a latency of less than $5 \mathrm{sec}$. The quantitative definition of a chain can be explored at different parameter values for the latency criterion, thereby providing insight into the utility of this parameter in describing the behavior.

Interpreting the data becomes more complicated with the added capabilities of measuring more than just the occurrence of an operant response (i.e., rate) and the addition of hopper entry variables. Not only is the investigator challenged with interpreting the dependent variables in relation to the independent variables, but he or she is also challenged with interpreting the relations among dependent variables. Exploration of the relationships among the dependent variables may help uncover putative motor, sensory, associative, or motivational differences that affect performance.

Sample data presented here suggest several hypotheses for further study. For example, individual differences were generally more prominent in the prefood than in the consumatory/reinforced-response period of the session. That is, individual differences in the disk press measures of rate and peak force were larger in the prefood periods than in the food-available period; similarly, the disk presshopper entry latency converged for the 3 mice in the food period. This suggests that the reinforcement contingency brought the behavior under control to the point that individual, and potentially genetic, differences might have been masked by environmental variables. Another interesting effect is the relatively excessive force and rate of responding of the CD-1 mouse during the prefood periods. Whether these results reflect heightened motivation to obtain food or an inability to learn that the stimulus conditions of Periods 1 and 2 specifically signaled unavailability of food must await further experimentation.

In regard to motor behavior and the force-time waveforms shown in Figure 1, the data suggest that the C57BL/6 mouse made responses of longer durations that had somewhat less narrowly defined peaks than those of the other 2 mice. This tendency toward lower force and response slowing was observed in a previous study that compared the same three strains in a lick task, which provided measures of the peak force, rhythm, and rate of licking (Wang $\&$ Fowler, 1999). In addition, recent data suggest that the C57BL/6 mouse is unusually susceptible to haloperidol's Parkinson-like tendency to interfere with movement initiation (Fowler, Zarcone, \& Vorontsova, in press). These observations, plus physiological data demonstrating lower indices of dopamine function in the C57BL/6 mice (Wang \& Fowler, 1999), suggest the hypothesis that response execution by the C57BL/6 mouse reflects Parkinson-like deficits, as compared with the BALB/c and CD1 mice. This is of considerable importance to behavioral genetics involving knockout mice, because the C57BL/6 mouse frequently serves as the background strain in which the targeted mutation is expressed (Gerlai, 1996). Under these circumstances, the behavioral deficits exhibited by knockout mice might be conditional on the presence of motor 
system abnormalities in the C57BL/6 background strain. Detailed, quantitative behavioral analyses, such as those afforded by the system described herein, should contribute to the analysis of this and other problems addressed in behavioral genetics with mice as the model organism.

\section{REFERENCES}

Crabbe, J. C., Wahlsten, D., \& Dudek, B. C. (1999). Genetics of mouse behavior: Interactions with laboratory environment. Science, 284, 1670-1672.

Fowler, S. C. (1974). A minicomputer system for recording the dynamic properties of individual operant responses. Behavior Research Methods \& Instrumentation, 6, 288-292.

Fowler, S. C. (1985). Amplitude measures of operant response: Implementation with Apple Pascal. Behavior Research Methods, Instruments, \& Computers, 17, 301-306.

Fowler, S. C. (1987). Force and duration of operant response as de- pendent variables in behavioral pharmacology. In T. Thompson, P. B. Dews, \& J. E. Barrett (Eds.), Neurobehavioral pharmacology: Advances in behavioral pharmacology (Vol. 6, pp. 83-127). Hillsdale, NJ: Erlbaum.

Fowler, S. C., \& Leberer, M. R. (1977). Hardware techniques for analog processing using the State Systems PDP-8 I/O interface. Behavior Research Methods \& Instrumentation, 9, 210-214.

Fowler, S. C., Zarcone, T. J., \& Vorontsova, E. (in press). Haloperidolinduced microcatalepsy differs in CD-1, BALB/c and C57BL/6 strains of mice. Experimental \& Clinical Psychopharmacology.

GERLAI, R. (1996). Gene-targeting studies of mammalian behavior: Is it the mutation or the background genotype? Trends in Neuroscience, 19, 177-181.

Notterman, J. M., \& Mintz, D. E. (1965). Dynamics of response. New York: Wiley.

SkINNER, B. F. (1938). The behavior of organisms. Acton, MA: Copley. WANG, G., \& FowLER, S. C. (1999). Effects of haloperidol and clozapine on tongue dynamics during licking in CD-1, BALB/c and C57BL/6 mice. Psychopharmacology, 147, 38-45.

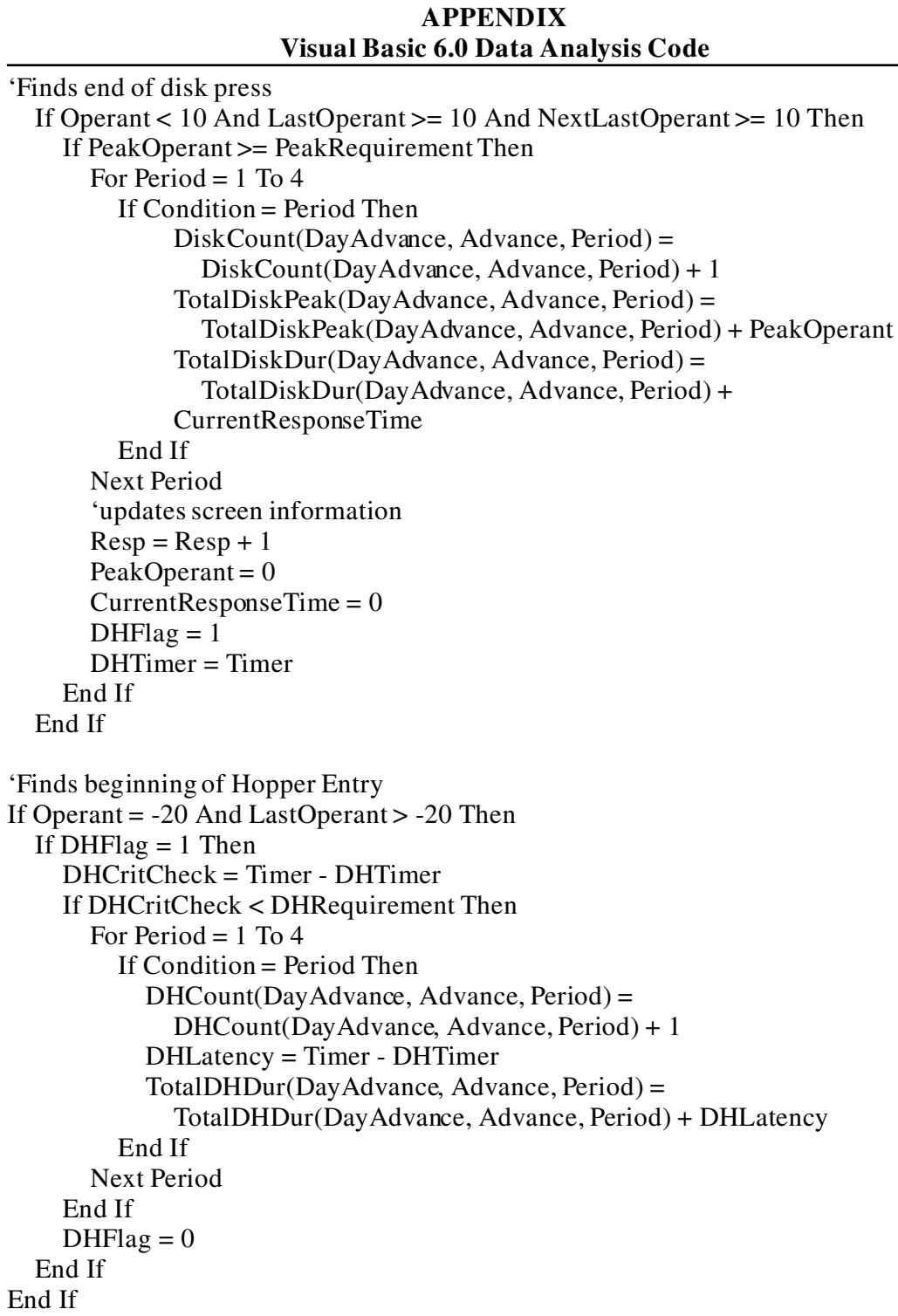


APPENDIX (Continued)

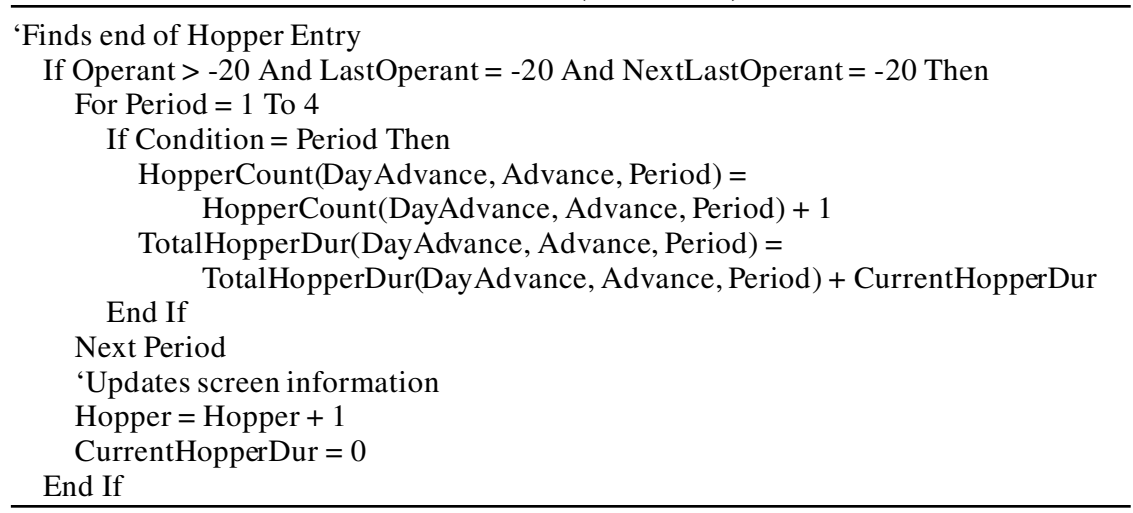

(Manuscript received October 2, 2000;

revision accepted for publication June 12, 2001.) 